\title{
Use of radiation from PC screen for non-destructive controlling the internal strains in transparent parts
}

\author{
V.P. Maslov, N.V. Kachur \\ V. Lashkaryov Institute of Semiconductor Physics, NAS of Ukraine, \\ 41, prospect Nauky, 03028 Kyiv, Ukraine
}

\begin{abstract}
It is known that the most widely used method to control internal strains is the optical polarization method. However, the sources of polarized radiation are the most problem issue of this method. There are some difficulties in using this method when testing large-scale optical parts, diameters of which exceed $500 \mathrm{~mm}$. To choose an optical source suitable for solving these tasks, we have analyzed the design of liquidcrystal screens and ascertained the possibility to use their polarized radiation in tests of internal strains in optical parts of large dimensions. Adduced below are the results of investigations of internal strains in parts of sapphire and organic glass.
\end{abstract}

Keywords: polarization method for non-destructive testing, radiation source, liquidcrystal screen, optical part.

Manuscript received 17.05.13; revised version received 14.07.13; accepted for publication 19.09.13; published online 30.09.13.

\section{Introduction}

It is known that internal strains in optically transparent parts and crystals influence essentially on functional performances and precision of optical devices [1-5]. Also, the polarization method is the most widely used for testing the internal strains in optically transparent materials $[6,7]$. Wikipedia describes this method in the following way.

The simplest manifestation of polarization is to visualize that a plane wave, which is a good approximation for light waves (a plane wave is a wave with infinitely long and wide wave front). For plane waves, Maxwell's equations, specifically the Gauss law, impose the transversality requirement that the electric and magnetic field should be perpendicular to the direction of propagation and to each other. Conventionally, when considering polarization, the electric field vector is described and the magnetic field is ignored, since it is perpendicular to the electric field and proportional to it. The electric field vector of a plane wave may be arbitrarily divided into two perpendicular components labeled $x$ and $y$ (with $z$ indicating the direction of travel). For a simple harmonic wave, where the amplitude of the electric vector varies in a sinusoidal manner in time, the two components have exactly the same frequency. However, these components have two other defining characteristics that can differ. First, the two components may not have the same amplitude. Second, the two components may not have the same phase, which means that they may not reach their maxima and minima at the same time. Mathematically, the electric field of a plane wave can be written as,

$\vec{E}(\vec{r}, t)=\operatorname{Re}\left[\left(A_{x}, A_{y} \cdot e^{i \phi}, 0\right) e^{i(k z-\omega t)}\right\rfloor$

or alternatively,

$\vec{E}(\vec{r}, t)=\left(A_{x} \cdot \cos (k z-\omega t), A_{y} \cdot \cos (k z-\omega t+\phi), 0\right)$,

where $A_{x}$ and $A_{y}$ are the amplitudes of the $x$ and $y$ directions and $\phi$ is the relative phase between these two components.

Internal strains change conditions for passing waves, which allows to get with an analyzer some visual pattern for internal strains over the whole area of the tested part.

For a long time, scientists and engineers have used this method not only for testing optical materials but investigating and optimizing the models for various building constructions (for instance, in designing bridges) made in small scales from organic glass. However, one of the problem issues in this method, there 
remains search and choice of the polarized light source. This source should:

- possess rather large area of radiation (comparable with that of the tested part);

- provide radiation uniform in its optical characteristics over the total area;

- be simple in operation;

- $\quad$ save the energy consumed.

Unfortunately, the devices for polarization control produced by industry possess an illuminator with the diameter not larger than $250 \mathrm{~mm}$, which prevents testing the large-scale parts. As to the latter, it is commonly adopted to consider optical parts with dimensions equal or larger than $500 \mathrm{~mm}$ as the large-scale ones.

Our investigations are aimed at searching new radiation sources of polarized light with linear dimensions larger than $250 \mathrm{~mm}$ to use them in testing internal mechanical strains in large-scale optical parts.

\section{Choosing a suitable light source}

In the process of searching, our attention was turned to a display screen of PC as a source of polarized light. Indeed, the construction of modern liquid-crystal screens, in accord with the work [8], always contains polarization plates or films.

Under action of an electric field, there takes place changing the structure of liquid crystals, which causes changes in conditions of polarized radiation passage from each pixel. The final role in image formation on the display is played by the polarizer placed directly before its external window. The polarization direction in this polarizer is shifted relatively to the first one (internal) by 90 degrees. Imaging the meaning of this external polarizer, we can conventionally extract it from the surface of the connected panel. In this case, we can see all the pixels illuminated up to their maximum, i.e., uniform white glowing from the whole screen independently of a displayed picture. The fact that light becomes polarized and its polarization plane is rotated by each cell differently (accordingly to the applied voltage) does not change something for our eyes. The function of the external polarizer just consists in separation of necessary wave components, which allows to see at the output the required image set by the computer.

Let us take for example a polarizer with the vertical direction of polarization, i.e., transmitting waves with the electrical vector oriented in the vertical plane.

Depicted in Fig. 1 is the wave propagating in the plane lying under some angle relatively to the vertical polarization direction. The electric vector of the incident wave can be expanded by two mutually orthogonal components: parallel to the polarizer optical axis and perpendicular to it. The first component, parallel to the optical axis, passes, while the second (perpendicular) one is blocked.

Thereof, it is obvious that two extreme positions exist: propagating waves with the only vertical polarization component will be transmitted without changes; and the wave propagating only in the horizontal plane will be fully blocked.

These two extreme positions correspond to absolutely open and absolutely closed cell states. Let us summarize:

- for maximum possible blocking the transmitting light by the cell (pixel), it is necessary for the polarization plane of this light to be orthogonal to the transmission plane (polarization direction) of the external polarizer;

- for the maximum possible transmission of the light, its polarization plane should coincide with the polarization direction.

Gradually regulating the voltage applied to the cell electrodes, one can control positions of liquid crystal molecules and, as a consequence, rotations of the polarization plane of the transmitted light. And due to it, one can change the amount of light transmitted through the cell.

As the rotation angle for the polarization plane depends on the distance covered by light in the layer of liquid crystals, this layer should possess the rigorously kept thickness over the whole panel surface. To provide uniformity in the distance between glasses (with all the structure deposited on them), the special spacers are applied.

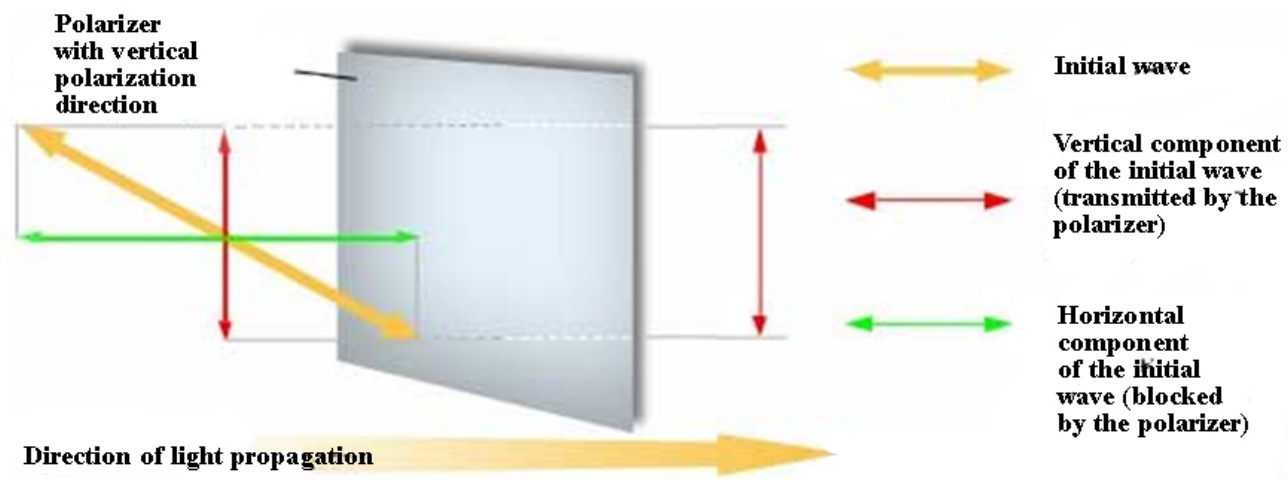

Fig. 1. Passage of light waves through the external polarizer. 


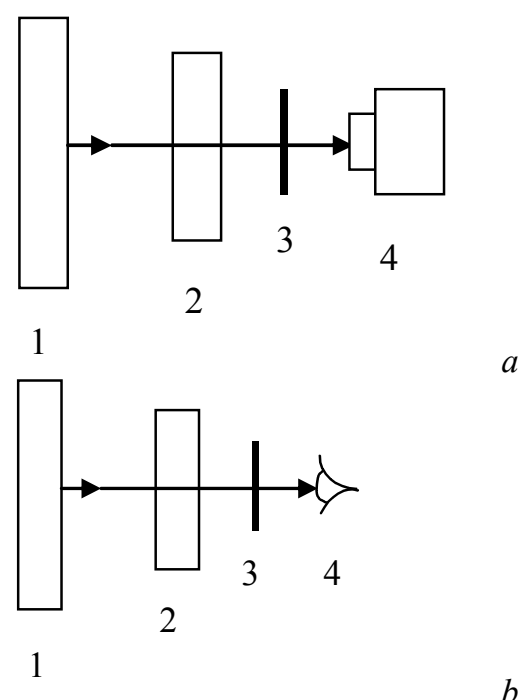

Fig. 2. Scheme for testing: (a) 1 - display screen; 2 - sample under investigation; 3 - analyzer; 4 - digital camera; (b) 4 visual observation.

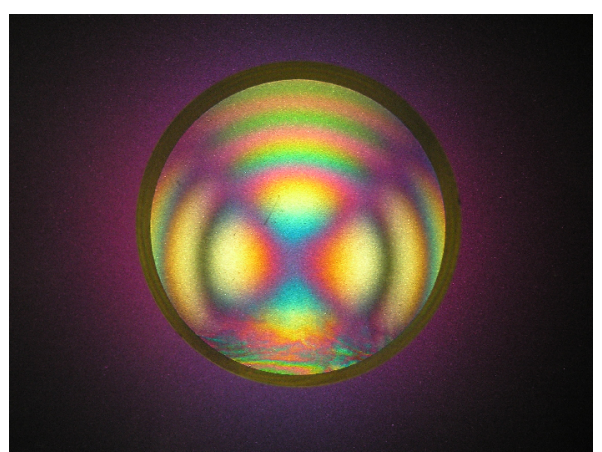

Fig. 3. Internal strains in the bottom part of the sapphire single crystal.

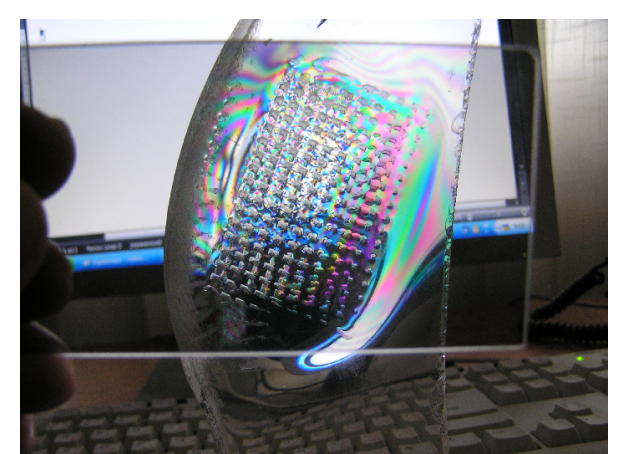

Fig. 4. Internal strains in organic glass after hot deformation.

Thus, radiation from the display screen is always linearly polarized. To provide a high brightness, using the computer we can set white background. Taking into account that the pixel size is close to $0.1 \ldots 0.3 \mathrm{~mm}$ in dependence of the needed resolution, radiation of polarized light is uniform over the screen area, and the screen dimensions of usual monitors reach 30 inches, i.e., $762 \mathrm{~mm}$, which fully satisfies testing even largescale optical parts.

Besides, it is known that the consumed power of this display screen do not exceed $40 \mathrm{~W}$. Thus, our choice of this economic source of polarized light seems to be promising for further investigations.

To test internal strains in optically transparent materials, we chose the traditional scheme (Fig. 2).

Our tests were performed using the samples made of two optically transparent materials, namely: sapphire and organic glass. As an analyzer, we used a polarization filter on the objective of digital camera, while for visual observations - polarization glasses. Figs 3 and 4 show defects in sapphire crystals and internal strains in organic glass.

To provide these tests in industrial conditions, it is desirable to have standards with preliminary set levels of internal strains. Then, the tests can be performed using comparison of images for the reference and tested samples.

\section{Conclusions}

Thus, the performed investigations have shown the possibility and perspectives for using liquid-crystalline screens of PC for non-destructive testing the internal strains in optically transparent parts.

\section{References}

1. G. Roebeen, M. Steen, J. Bressers, O. Van der Biest, Mechanical fatigue in monolithic nontranforming ceramics // Progr. in Mater. Sci. 40, p. 265-331 (1996).

2. Strength of Optical Glasses. - The Schott Glass Company.

3. J.C. Lambropoulos, S.R. Arrasmith, S.D. Jacobs, D. Golini, Manufacturing-induced residual stresses in optical glasses and crystals: Example of residual stress relief by magnetorheological finishing (MRF) in commercial silicon wafers // Proc. SPIE, 4451, Optical Manufacturing and Testing, IV, p. 181 (2001).

4. V. Genberg, G. Michels, K. Doyle, Making mechanical FEA results useful in optical design // Proc. SPIE, 4761 (2002).

5. K.B. Doyle, J.M. Hoffman, V.L. Genberg, G.J. Michels, Stress birefringence modeling for lens design and photonics // Intern. Opt. Design Conf., Tucson, Arizona, 2002.

6. Polarized Light, Ed. D. Goldstein, Second edition. Marcel Dekker, Inc., New York, 2003.

7. E. Collett, Field guide to polarization // SPIE Field Guides, FG05, 2005.

8. Hirohisa Kawamoto, The history of liquid-crystal displays // Proc. IEEE, 90(4), p. 460-500 (2002).

9. Patent 78911, Ukraine, G02F1/13. Application of plane or TV screen in the state of connection to the electric power supply of PC or TV set as a source of polarized emission / Ye.F. Venger, V.P. Maslov, O.I. Semenets', N.V. Kachur, S.M. Kushchovyi. Publ. 10.04.2013, Bulletin № 7/2013. 